\title{
An Inverted Magnetron Operating in HiPIMS Mode
}

\author{
Phitsanu Poolcharuansin ${ }^{1,2, *} \mathbb{C}$, Artit Chingsungnoen ${ }^{1,2}$, Nitisak Pasaja ${ }^{1,2}$ and \\ James W Bradley ${ }^{3}$ \\ 1 Technological Plasma Research Unit, Department of Physics, Faculty of Science, Mahasarakham University, \\ Maha Sarakham 44150, Thailand; artit.c@msu.ac.th (A.C.); nitisak.p@msu.ac.th (N.P.) \\ 2 Thailand Center of Excellence in Physics, Commission on Higher Education, 328 Si Ayutthaya Road, \\ Bangkok 10400, Thailand \\ 3 Department of Electrical Engineering and Electronics, University of Liverpool, Brownlow Hill, \\ Liverpool L69 3GJ, UK; J.W.Bradley@liverpool.ac.uk \\ * Correspondence: phitsanu.p@msu.ac.th; Tel.: +66-4375-4379
}

Received: 1 November 2018; Accepted: 26 November 2018; Published: 27 November 2018

\begin{abstract}
An ionized physical vapor deposition technique for thin ferromagnetic films is proposed. The technique is based on high power impulse magnetron sputtering (HiPIMS) with positive discharge polarity. A gapped-target was employed as the cathode of the magnetron. By applying positive HiPIMS pulses to the anode, sputtered particles inside the magnetron source were ionized and extracted through the gap. Using a discharge current with a peak of about $13 \mathrm{~A}$, an ion flux in the order of $10^{21} \mathrm{~m}^{-2} \mathrm{~s}^{-1}$ was obtained at a distance of $45 \mathrm{~mm}$ from the magnetron. In addition, deposition rates of up to $1.1 \AA / \mathrm{s}$ for nickel films were achieved using a $30 \mathrm{~Hz}$ repetition rate and $300 \mu$ s pulse width.
\end{abstract}

Keywords: ionized physical vapor deposition; magnetron sputtering; HiPIMS; ferromagnetic thin films

\section{Introduction}

In conventional magnetron sputtering, energetic electrons are effectively confined due to the presence of transverse magnetic field lines above the target surface. As a consequence, both the ionization rate of the sputtering gas and the sputtering rate of the target material are significantly increased [1].

This is not the case for ferromagnetic metals, e.g., nickel, iron, and cobalt. A ferromagnetic target performs as a magnetic field conductor, completing the magnetic circuit of the magnetron. As a result, most of the magnetic field lines above the target are absent. Therefore, the conventional design of the magnetron is not suitable for the deposition of ferromagnetic films.

Since ferromagnetic thin films are widely used in industrial applications [2,3], a number of magnetron configurations have been proposed that deposit at an acceptable deposition rate [4-7]. However, the planar Penning trapping configuration [8] is of particular interest here. In this configuration, a circular plate and a concentric ring with a ferromagnetic target are used to form an annular narrow gap. The gap allows some leaking of magnetic field lines and provides the transverse component of the field. The anode cavity below the gap is also introduced to prevent sputtering of the target backing plate.

Recently, the inverted gapped-target magnetron was developed as a device for ferromagnetic film deposition, providing a means to produce good quality, crack-free nickel films [9]. This is mainly due to the ion bombardment with energy of about $200 \mathrm{eV}$ during the film growth. However, the ion density (as implied from the measured discharge current) is rather low when operated in direct current 
(DC) mode. In addition, the majority of the energetic ions are ionized from the sputtering gas, e.g., argon ions.

High power impulse magnetron sputtering (HiPIMS) is now a well-established technique to generate large ion fluxes of sputtered particles [10]. By applying pulses of high discharge power to a magnetron target, a considerable amount of sputtered species can be ionized by electron-impact ionization in the dense plasma.

The main purpose of the present work was to apply the HiPIMS technique to the inverted magnetron in order to enhance the density of the energetic ions of both gas and metal species. This requires the further modification of a HiPIMS power supply to drive the inverted magnetron with positive pulsed voltages. The discharge characteristics of the magnetron operated in the HiPIMS mode were investigated and are discussed. In addition, the relative deposition rates and ion flux for a number of process conditions were measured using a quartz crystal microbalance $(\mathrm{QCM})$ and a planar electrical probe, respectively.

\section{Materials and Methods}

The structure of the inverted magnetron has been provided in detail elsewhere [9]. However, a schematic diagram showing the arrangement of the magnetron used in the present work is shown in Figure 1a. The magnetron mainly consisted of a 410-grade stainless-steel anode and a gapped-nickel cathode. The anode was biased to the positive driven-voltage, while the cathode was grounded. The gapped-nickel cathode consisted of two concentric pieces, i.e., a central disk and a ring. The diameter of the central disk was about $31 \mathrm{~mm}$, while the inner and the outer diameter of the ring target was $37 \mathrm{~mm}$ and $70 \mathrm{~mm}$, respectively. The thickness of both the central disk and the ring was about $6 \mathrm{~mm}$. Using this target configuration, a complete circular gap was formed with a width of $3 \mathrm{~mm}$. It is important to note that the central disk was actually supported by a target holder held at the axis of the magnetron. However, the holder of the central disk is not shown in Figure 1a for simplicity. The permanent magnets embedded in the anode were employed to generate the magnetic field for the electron confinement at the cathode gap. As a result, the plasma was able to be generated around the cathode gap, enabling the sputtering of cathode material.

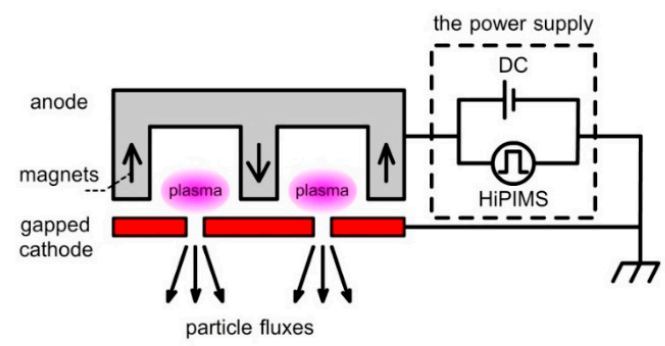

(a)

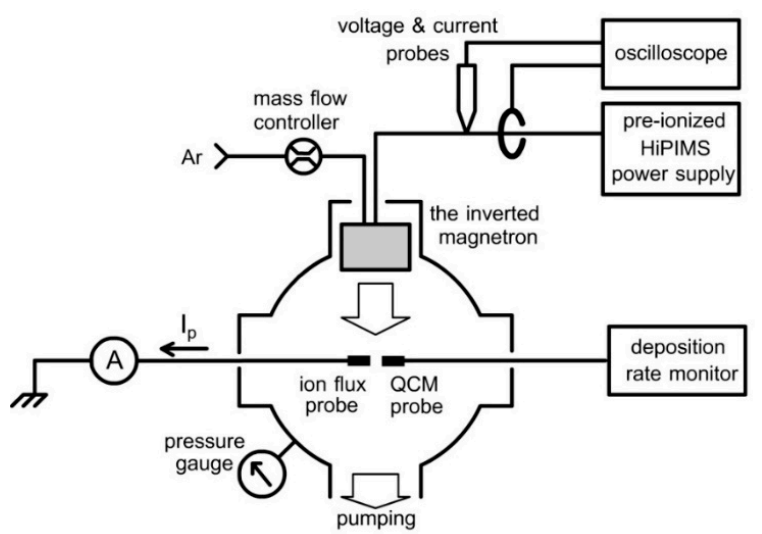

(b)

Figure 1. Schematic diagram of (a) the inverted magnetron and (b) experimental arrangements. 
Power was supplied to the plasma using a DC pre-ionizer coupled to a HiPIMS unit, in principle, similar to that used in a previous report [11], but with opposite polarity. The DC unit was used to ignite and sustain the background plasma, while the main discharge power was delivered by the HiPIMS unit. The width $(w)$ and repetition rate $(f)$ of the HiPIMS pulse can be adjusted in the range of $20-400 \mu$ s and $10-500 \mathrm{~Hz}$, respectively. However, a pulse repetition rate of $30 \mathrm{~Hz}$ and a pulse width of $300 \mu$ s were selected as the HiPIMS pulse parameters for the present work.

The inverted magnetron was installed in an in-house coating system, as shown in Figure $1 \mathrm{~b}$. A cylindrical vacuum chamber was evacuated to a base pressure of $5 \times 10^{-4} \mathrm{~Pa}$ by a diffusion pump and a rotary pump. Argon as the sputtering gas was introduced to the chamber via a mass flow controller (4850, Brooks Instrument, Hatfield, PA, country) at a flow rate between $10 \mathrm{sccm}$ and $50 \mathrm{sccm}$. As a result, the process pressure between $0.3 \mathrm{~Pa}$ and $13 \mathrm{~Pa}$ could be maintained. In a routine operation, the background plasma is initially ignited at a rather high pressure using the positive DC unit. Then, the argon flow rate is reduced to establish a designed process pressure before applying positive HiPIMS pulses to the magnetron. Using this approach, HiPIMS plasma can be generated during the pulse at pressures down to about $1 \mathrm{~Pa}$.

The waveforms of the discharge voltage $\left(V_{d}\right)$ and current $\left(I_{d}\right)$ were measured using a high voltage probe (10076B, Agilent, Santa Clara, CA, USA) and a non-invasive current monitor (101, Pearson Electronics, Palo Alto, CA, USA), respectively. A digital oscilloscope (DSO1002A, Agilent, Santa Clara, CA, USA) were employed for the monitoring and recording of the $V_{d}$ and $I_{d}$ waveforms. The waveforms of the discharge power $\left(P_{d}\right)$ can be obtained from $P_{d}=V_{d} I_{d}$.

To investigate the effect of the average discharge power on the particle fluxes arriving at a typical substrate position, probing was performed using: (1) a planar electrical probe to determine the ion flux, and (2) a QCM to determine the sputtered particle arrival rates. The planar probe had a circular stainless steel exposed surface of $16 \mathrm{~mm}$ diameter and was grounded to obtain a saturation current $\left(I_{p}\right)$, monitored by a current probe (2877, Pearson Electronics, Palo Alto, CA, USA). Hence, the ion fluxes arriving to the probe could be easily determined. The deposition rate and thickness of the formed nickel films was determined in the vicinity of the flux probe using the QCM devices (STM-2XM, Inficon, Badragaz, Switzerland), both $45 \mathrm{~mm}$ from the cathode gap.

\section{Results}

The discharge waveforms for a process pressure of $1 \mathrm{~Pa}$ are shown in Figure 2. In general, the waveforms were almost the same as those of a conventional HiPIMS except for the polarity. In the case of the inverted configuration, the discharge signals were all positive with respect to the ground.

The background plasma, $P_{D C}$, required a low $\mathrm{DC}$ power of about $70 \mathrm{~W}$ with a sustained voltage and current of about $230 \mathrm{~V}$ and $0.3 \mathrm{~A}$, respectively. The discharge changed to high power mode when a positive pulse from the HiPIMS unit was applied to the anode. Using the initial HiPIMS voltage of $540 \mathrm{~V}$ at $t=0 \mu \mathrm{s}$, a discharge current of up to a peak $\left(I_{\text {peak }}\right)$ of $13 \mathrm{~A}$ developed during the on-time phase $(t=0-300 \mu \mathrm{s})$, yielding a peak discharge power $\left(P_{\text {peak }}\right)$ of about $5 \mathrm{~kW}$ (see Figure $\left.2 \mathrm{a}-\mathrm{c}\right)$. It is worth mentioning that the $V_{d}$ sharply dropped to below $400 \mathrm{~V}$, corresponding to the high discharge current drawn to the magnetron. The discharge current waveform generally indicates the variation of the plasma density [12]. The discharge current jumped from $~ 0.3 \mathrm{~A}$ in DC mode to $13 \mathrm{~A}$ in HiPIMS mode. The density of the plasma in DC mode has been reported to be approximately $0.6 \times 10^{16} \mathrm{~m}^{-3}$ [9]. Therefore, a plasma density during the HiPIMS pulses in the order of $3 \times 10^{16} \mathrm{~m}^{-3}$ could be expected.

The high discharge current during the HiPIMS pulse agreed well with the probe current determining the ion fluxes at the probe position. The current drawn to the grounded probe is plotted in Figure 3. In the high power mode, an $\mathrm{I}_{\mathrm{p}}$ with a peak value of $0.06 \mathrm{~A}$ was obtained at the end of the pulse $(\mathrm{t} \sim 300 \mu \mathrm{s})$. It is worth mentioning that a positive current of several hundreds of microamperes was observed even in the DC mode. The positive probe current observed during both the DC and HiPIMS mode indicated that the ion flux arriving to the grounded probe was significantly larger than the electron flux. 

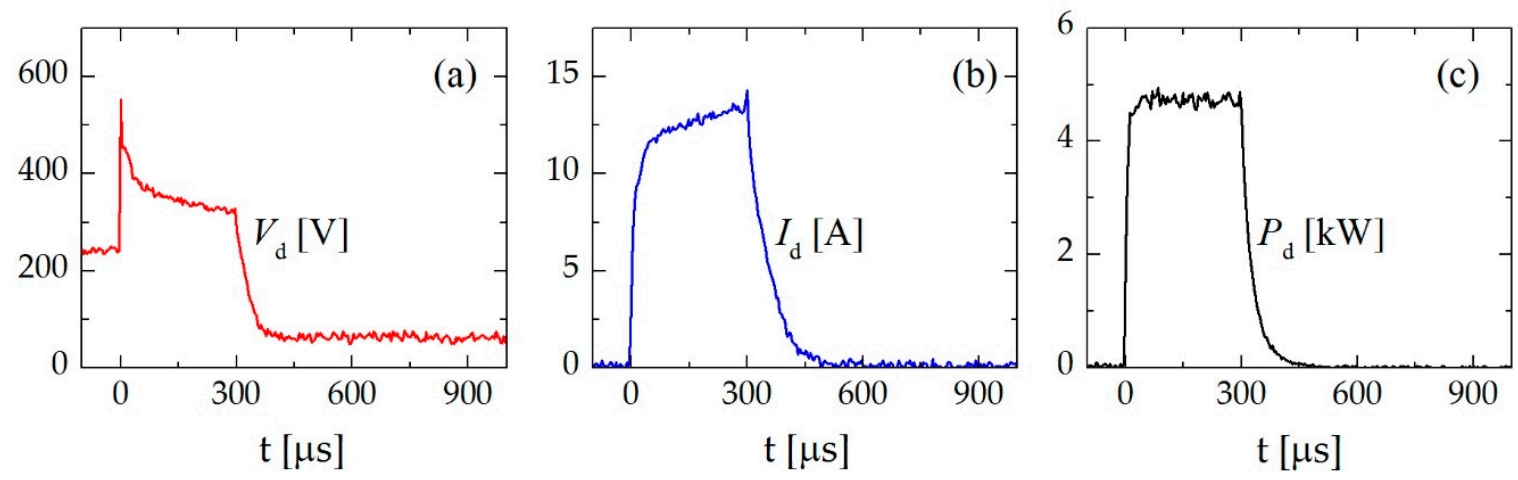

Figure 2. Waveforms of (a) the discharge voltage, (b) the discharge current and (c) the discharge power at the process pressure of $1 \mathrm{~Pa}$. The onset of the pulse is at $t=0 \mu \mathrm{s}$.

It is highly possible that the large ion flux to the grounded probe is mainly due to a high voltage plasma sheath present above the probe surface. The plasma sheath is a layer of electric field formed owing to the potential difference between the plasma potential and the probe potential [13]. Using a Langmuir probe, a plasma potential of larger than $120 \mathrm{~V}$ has been estimated in the DC mode [9], causing electron suppression to the grounded probe. Therefore, the electron flux to the probe can be negligible compared to the ion fluxes. In other words, the measured probe current can be used to represent the ion flux $\left(\Gamma_{i}\right)$ to the probe as $\Gamma_{i} \sim I_{p} /\left(q_{i} A_{p}\right)$, where $q_{i}$ is the average charge of the ion species (mostly singly charged ions) which is $1.6 \times 10^{-19} \mathrm{C}$ and $A_{p}$ the probe area.

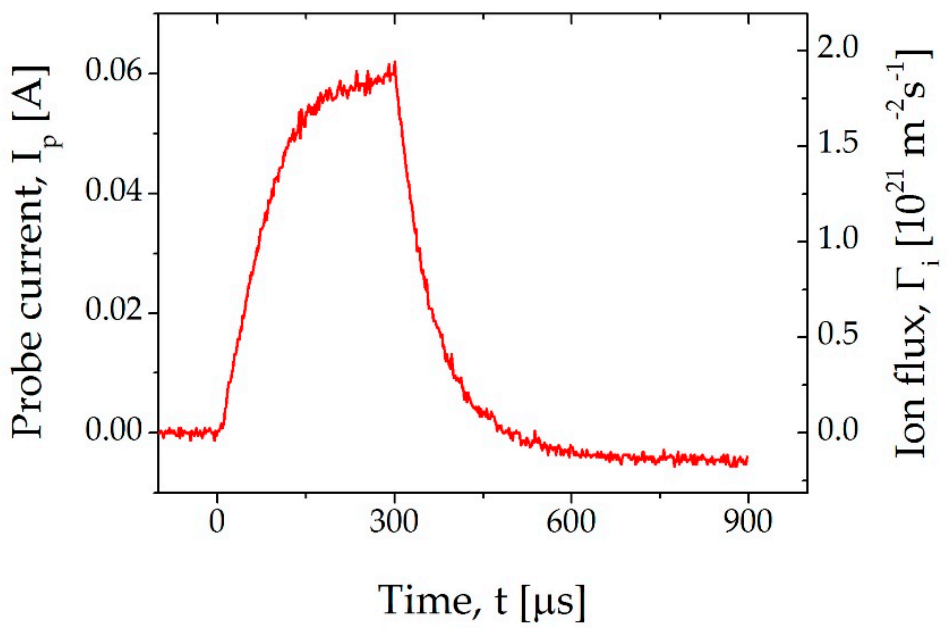

Figure 3. The temporal evolution of the probe current and the corresponding ion flux arriving to the grounded probe.

The corresponding ion flux, calculated from the probe current, is also shown in Figure 3. The ion flux during the HiPIMS pulse dramatically increased to a peak of $\Gamma_{i \text {,peak }} \sim 10^{21} \mathrm{~m}^{-2} \mathrm{~s}^{-1}$. It was two orders of magnitude higher than the ion flux of $\sim 10^{19} \mathrm{~m}^{-2} \mathrm{~s}^{-1}$ during the DC mode [9].

Figure 4 shows the peak ion flux $\Gamma_{i, p e a k}$ determined near the pulse terminal $(t \sim 250-300 \mu \mathrm{s})$ as a function of the peak of the discharge current $I_{d \text {,peak }}$. It can be seen that the $\Gamma_{i, p e a k}$ was mostly proportional to the $I_{d \text {,peak }}$, especially in the high current range. The increase of the ion flux mainly contributed to the large discharge current, as well as the discharge power generating the dense plasma during the HiPIMS mode.

The dense plasma increased not only the ion flux but also the sputtered particles. More importantly, the sputtered nickel particles could be effectively ionized in the plasma and could be the major contributor to the thin film deposition process. 


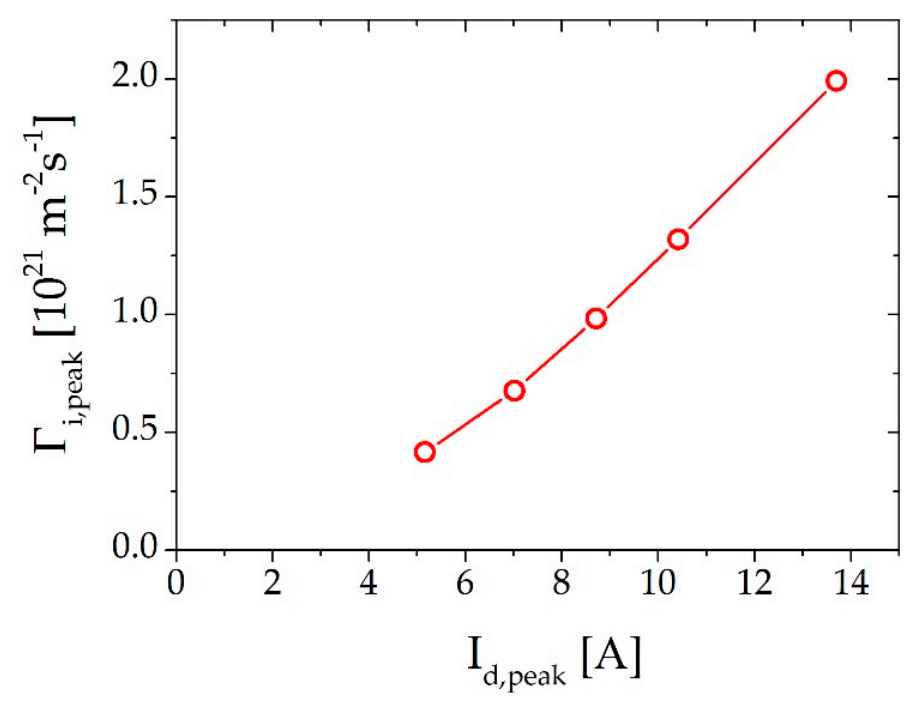

Figure 4. The peak of ion flux $\Gamma_{i, p e a k}$ as a function of the peak of the discharge current $I_{d \text {,peak }}$.

Deposition rates $\left(R_{d}\right)$ of nickel films obtained from the QCM reading as a function of the average discharge power $\left(P_{d, a v g}\right)$ are shown in Figure 5 . It is worth mentioning that the average discharge power was calculated using the equation $P_{d, a v g}=f \int_{0}^{w} P_{d} d t+P_{D C}$. The deposition rate tends to increase with the average HiPIMS power. The maximum deposition rate for the given process condition was about $1.1 \AA / \mathrm{s}$.

In a conventional HiPIMS discharge, during which the negative driving voltage is applied to the target, the plot between the deposition rate and the discharge power density shows a less-than-linear curve [14]. This is due to the returning effect, where the positive metal-ions return to the target, decreasing the deposition rate, particularly at the high discharge power level [15]. This may be not the case for the inverted magnetron. The metal ions created in the inverted magnetron can readily be extracted to the substrate using the positive driving voltage. As a result, a linear deposition rate curve was observed, as seen in Figure 5.

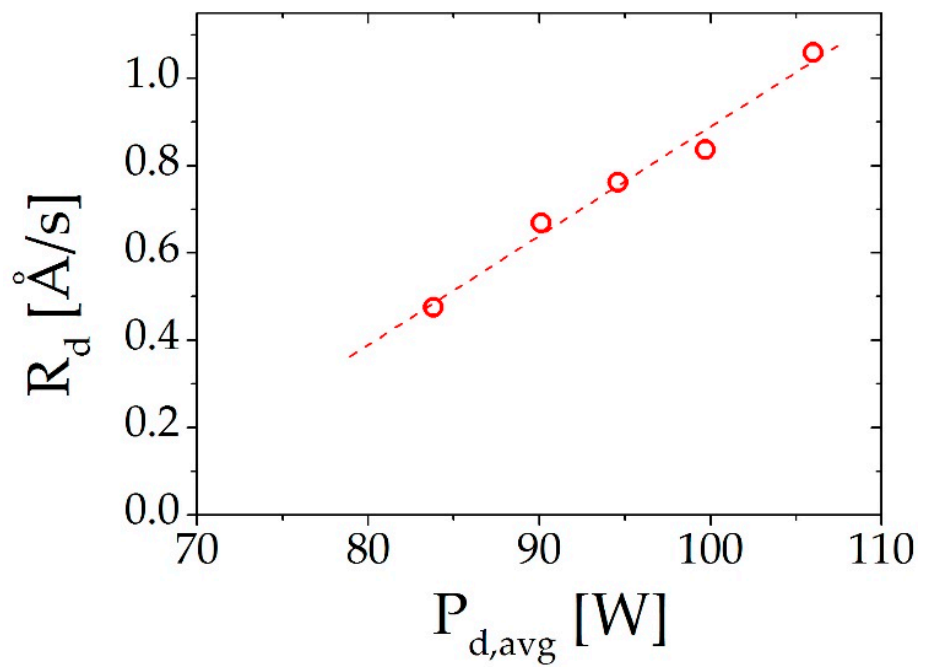

Figure 5. Deposition rate as a function of the discharge power density for $30 \mathrm{~Hz}$ with $300 \mu \mathrm{s}$ pulse parameters.

It has been pointed out that the flakes of accumulated film in the magnetron could cause a short-circuit between the cathode and the anode [8]. However, by operating the inverted magnetron in the HiPIMS mode, the amount of the flakes in the magnetron could possibly be reduced, due to ionization and the extraction of the sputtered particles. 


\section{Discussion}

It is reasonable to assume that the discharge mostly occurs inside the magnetron. Therefore, the target surface adjacent to the plasma could be sputtered. In the DC mode, sputtered particles (e.g., Ni) could either be deposited on the anode surface or leave the magnetron through the cathode gap along with the $\mathrm{Ar}^{+}$ions. In the HiPIMS mode, the plasma density inside the magnetron was expected to be high. Considerable amounts of sputtered particles in the magnetron can be ionized, (e.g., $\mathrm{Ni}^{+}$ and $\mathrm{Ni}^{2+}$ ), and extracted through the gap forming metal-rich plasma to the substrate.

Using the ion flux probe together with the QCM probe allows us to understand the conceptual idea as follows. The total ion current, obtained from the electrically grounded probe, is proportional to the metal ion flux $\left(\Gamma_{m i}\right)$ and gas ion flux $\left(\Gamma_{g i}\right)$, written as:

$$
I_{i} \propto \Gamma_{m i}+\Gamma_{g i}
$$

Or

$$
I_{i}=q_{i} A_{p}\left(\Gamma_{m i}+\Gamma_{g i}\right)
$$

where $q_{i}$ and $A_{p}$ are the elementary charge on the ion and the probe area, respectively.

In the case of QCM, the deposition rate is proportional to the combination between the metal ion flux $\left(\Gamma_{m i}\right)$ and the metal neutral flux $\left(\Gamma_{m n}\right)$, written as:

$$
R_{d} \propto\left(\Gamma_{m i}+\Gamma_{m n}\right)
$$

Or

$$
R_{d}=K\left(\Gamma_{m i}+\Gamma_{m n}\right),
$$

where $K$ is a constant related to the specific process conditions, e.g., the actual density of the depositing film, the target to substrate distance, working pressure, etc.

Since $K$ in Equation (4) is difficult to determine, we focus attention on the relations Equations (1) and (3). The ratio between total ion current, $I_{i}$, and deposition rate, $R_{d}$, for the HiPIMS mode could be written as:

$$
\frac{I_{i}}{R_{d}} \propto\left(\frac{\Gamma_{m i}}{\Gamma_{m i}+\Gamma_{m n}}\right)+\left(\frac{\Gamma_{g i}}{\Gamma_{m i}+\Gamma_{m n}}\right)
$$

The ratio $I_{i} / R_{d}$ is proportional to the summation of two terms. The first term is the ionized metal flux fraction, $\Theta_{m}=\Gamma_{m i} /\left(\Gamma_{m i}+\Gamma_{m n}\right)$ [16]. The second term is the ionized gas flux to total metal flux fraction, $\Theta_{g}=\Gamma_{g i} /\left(\Gamma_{m i}+\Gamma_{m n}\right)$.

Figure 6 shows $I_{i} / R_{d}$ as a function of the average discharge power operating in the DC mode and the HiPIMS mode. The ratio of $I_{i} / R_{d}$ during the DC mode remains nearly constant. This ratio, however, significantly increases when applying the HiPIMS pulses to the magnetron.

The general trends of $I_{i} / R_{d}$ shown in Figure 6 agree well with Equation (5). In the DC mode, the $\Gamma_{m i}$ to the probes can be negligible, owing to the low plasma density. In this case, the $I_{i} / R_{d}$ ratio is proportional only to $\Gamma_{g i} / \Gamma_{m n}$. Even though increasing the DC power may increase both $\Gamma_{g i}$ and $\Gamma_{m n}$, the measured $I_{i} / R_{d}$ ratio is observed to stay nearly constant for the given range of the DC discharge power. In contrast, in the HiPIMS discharge mode with a peak power of a few $\mathrm{kW}, \Gamma_{m i}$ and the consequent $\Theta_{m}$ can significantly increase. This is mainly due to the enhancement of the ionization of nickel atoms in the high density plasma. The ratio of $I_{i} / R_{d}$ in the probe position is then observed to increase with the increasing average power.

Operating the magnetron in the HiPIMS mode enhances not only the ionized metal flux fraction $\Theta_{m}$ at the probe position, but also the density of energetic $\mathrm{Ni}^{+}$ions (and high ionization states). In our previous work [9], it was shown that the most probable kinetic energy, $E_{m}$, of ions extracted from the magnetron is closely related to the discharge voltage, $V_{d}$. Using the data in [9], we can roughly estimate that $E_{m} \sim 0.7 q V_{d}$ ( $q$ is the elementary charge). In the present work, $V_{d}$ during the HiPIMS 
pulse is about $350 \mathrm{~V}$ (see Figure 2a). Therefore, the $E_{m} \mathrm{of} \mathrm{Ni}^{+}$is approximately $240 \mathrm{eV}$, corresponding to the ion speed of $2.8 \times 10^{4} \mathrm{~m} / \mathrm{s}$. Using the measured flux of $1.7 \times 10^{21} \mathrm{~m}^{-2} \mathrm{~s}^{-1}$ (see Figure 3), the density of $\mathrm{Ni}^{+}$during the HiPIMS pulse was estimated to be approximately $6.0 \times 10^{16} \mathrm{~m}^{-3}$.

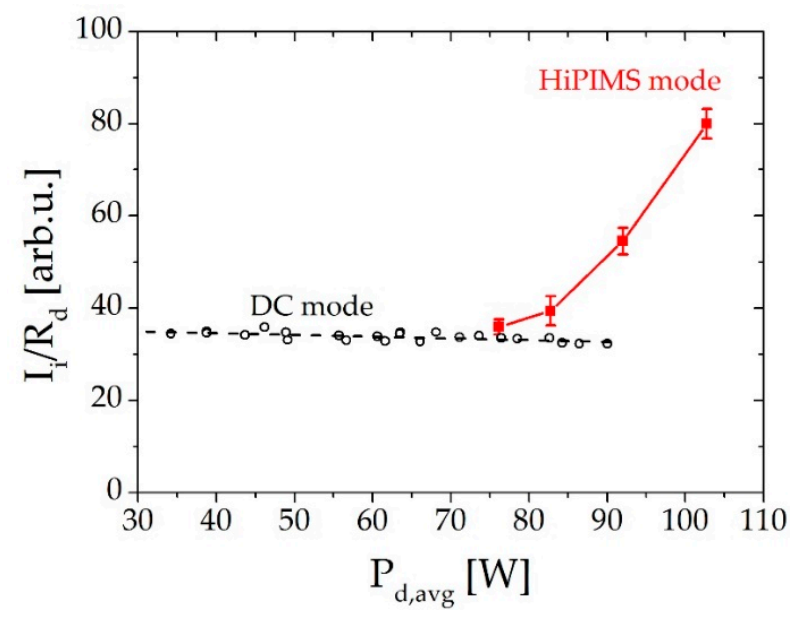

Figure 6. The ratio of the probe current, $I_{i}$, to the deposition rate, $R_{d}$, as a function of the average discharge power, $P_{d, a v g}$, delivered to the magnetron.

Despite the high density of the energetic $\mathrm{Ni}^{+}$ions, the performance of the gapped magnetron can be discussed as follows. The target gap is similar to the racetrack of the conventional target, where most electrons are effectively confined. In the gapped configuration, the target erosion mostly occurs near the edge of the target gap. We can expect that the gap width will be progressively wider for long-term operation. Furthermore, the discrete erosion may cause an additional instability to the discharge, in particular, when operated in the HiPIMS mode [17]. Further investigation needs to systematically and carefully be performed in order to validate the gapped configuration, both in term of plasma and thin film characterization.

\section{Conclusions}

An inverted magnetron operating in a high power impulse magnetron sputtering (HiPIMS) mode was proposed. The magnetron is electrically arranged as an inverted magnetron source in which the discharge is sustained using the positive driving voltage. This configuration could provide a large flux of energetic ions, assisting the thin film process. During the positive-voltage HiPIMS pulses, an ion flux in the order of $10^{21} \mathrm{~m}^{-2} \mathrm{~s}^{-1}$ and with a density of $6.0 \times 10^{16} \mathrm{~m}^{-3}$ was achieved. Using the ratio between the ion flux and deposition rate, it was interpreted that the ionized metal flux fraction during the HiPIMS mode was significantly higher than that during the DC mode. The linear relationship between the deposition rate and the discharge power indicated little or no back-attraction of ions to the cathode, which is not the case in a conventional HiPIMS.

Author Contributions: Conceptualization, P.P. and J.WB.; methodology, P.P., A.C. and N.P.; formal analysis, P.P., A.C. and N.P.; investigation, P.P., A.C. and N.P.; writing-original draft preparation, P.P.; writing-review and editing, P.P. and J.WB.

Funding: This research was funded by Thailand Center of Excellence in Physics (ThEP) and Faculty of Science, Mahasarakham University, grant number ThEP-61-EQP-MSU2.

Acknowledgments: The authors would like to thank the Integrated Nanotechnology Research Center, Khon Kaen University for technical support of a number of coating equipment.

Conflicts of Interest: The authors declare no conflict of interest. 


\section{References}

1. Abolmasov, S.N. Physics and engineering of crossed-field discharge devices. Plasma Sources Sci. Technol. 2012, 21, 035006. [CrossRef]

2. Howard, J.K. Thin films for magnetic recording technology: A review. J. Vaccum Sci. Technol. A 1986, 4, 1-13. [CrossRef]

3. Qiu, J.; Tang, X.S.; Chen, H.J.; Liu, X.; Hu, Z.W. A tunable broadband magnetoelectric and electromagnetic hybrid vibration energy harvester based on nanocrystalline soft magnetic film. Surf. Coat. Technol. 2017, 320, 447-451. [CrossRef]

4. Keraudy, J.; Delfour-Peyrethon, B.; Ferrec, A.; Molleja, J.G.; Richard-Plouet, M.; Payen, C.; Hamon, J.; Corraze, B.; Goullet, A.; Jouan, P.Y. Process- and optoelectronic-control of NiOx thin films deposited by reactive high power impulse magnetron sputtering. J. Appl. Phys. 2017, 121. [CrossRef]

5. Loch, D.A.L.; Gonzalvo, Y.A.; Ehiasarian, A.P. Nickel coatings by Inductively Coupled Impulse Sputtering (ICIS). Surf. Coat. Technol. 2015, 267, 98-104. [CrossRef]

6. Potocnik, J.; Nenadovic, M.; Bundaleski, N.; Jokic, B.; Mitric, M.; Popovic, M.; Rakocevice, Z. The influence of thickness on magnetic properties of nanostructured nickel thin films obtained by GLAD technique. Mater. Res. Bull. 2016, 84, 455-461. [CrossRef]

7. Caillard, A.; El'Mokh, M.; Semmar, N.; Dussart, R.; Lecas, T.; Thomann, A.L. Energy Transferred From a Hot Nickel Target During Magnetron Sputtering. IEEE Ttrans. Plasma Sci. 2014, 42, 2802-2803. [CrossRef]

8. Window, B.; Sharples, F. Magnetron sputtering sources for ferromagnetic material. J. Vaccum Sci. Technol. A 1985, 3, 10-13. [CrossRef]

9. Poolcharuansin, P.; Laokul, P.; Pasaja, N.; Chingsungnoen, A.; Horprathum, M.; Chindaudom, P.; Bradley, J.W. An inverted gapped-target sputter magnetron for the deposition of thin ferromagnetic films. Vacuum 2017, 141, 41-48. [CrossRef]

10. Gudmundsson, J.T.; Brenning, N.; Lundin, D.; Helmersson, U. High power impulse magnetron sputtering discharge. J. Vaccum Sci. Technol. A 2012, 30, 030801. [CrossRef]

11. Poolcharuansin, P.; Liebig, B.; Bradley, J. Plasma Parameters in a Pre-Ionized HiPIMS Discharge Operating at Low Pressure. IEEE Trans. Plasma Sci. 2010, 38, 3007-3015. [CrossRef]

12. Poolcharuansin, P.; Bradley, J.W. Short- and long-term plasma phenomena in a HiPIMS discharge. Plasma Sources Sci. Technol. 2010, 19, 025010. [CrossRef]

13. Lieberman, M.A.; Lichtenberg, A.J. Principles of Plasma Discharges and Materials Processing; Wiley: Hoboken, NJ, USA, 2005.

14. Hala, M.; Capek, J.; Zabeida, O.; Klemberg-Sapieha, J.E.; Martinu, L. Pulse management in high power pulsed magnetron sputtering of niobium. Surf. Coat. Technol. 2012, 206, 4186-4193. [CrossRef]

15. Anders, A. Deposition rates of high power impulse magnetron sputtering: Physics and economics. J. Vaccum Sci. Technol. A 2010, 28, 783-790. [CrossRef]

16. Poolcharuansin, P.; Bowes, M.; Petty, T.J.; Bradley, J.W. Ionized metal flux fraction measurements in HiPIMS discharges. J. Phys. D Appl. Phys. 2012, 45. [CrossRef]

17. Hecimovic, A.; von Keudell, A. Spokes in high power impulse magnetron sputtering plasmas. J. Phys. D Appl. Phys. 2018, 51. [CrossRef]

(c) 2018 by the authors. Licensee MDPI, Basel, Switzerland. This article is an open access article distributed under the terms and conditions of the Creative Commons Attribution (CC BY) license (http://creativecommons.org/licenses/by/4.0/). 